\title{
THE TOP LEFT DERIVED FUNCTORS OF THE GENERALISED I-ADIC COMPLETION
}

\author{
TRAN TUAN NAM
}

(Received 15 August 2012; accepted 11 September 2012; first published online 4 January 2013)

\begin{abstract}
We study the top left derived functors of the generalised $I$-adic completion and obtain equivalent properties concerning the vanishing or nonvanishing of the modules $L_{i} \Lambda_{I}(M, N)$. We also obtain some results for the sets $\operatorname{Coass}\left(L_{i} \Lambda_{I}(M ; N)\right)$ and $\operatorname{Cosupp}_{R}\left(H_{i}^{I}(M ; N)\right)$.
\end{abstract}

2010 Mathematics subject classification: primary 13D45; secondary 16E30; 13J99.

Keywords and phrases: derived functor, generalised I-adic completion, (generalised) local homology, co-associated prime.

\section{Introduction}

Let $R$ be a noetherian commutative ring and $I$ an ideal of $R$. In [10] the generalised $I$-adic completion $\Lambda_{I}(M, N)$ of the $R$-modules $M, N$ is defined by

$$
\Lambda_{I}(M, N)=\lim _{t}^{\lim }\left(M / I^{t} M \otimes_{R} N\right)
$$

When $M=R$, we have $\Lambda_{I}(R, N) \cong \Lambda_{I}(N)$, the $I$-adic completion of $N$. For each $R$-module $M$, there is a covariant functor $\Lambda_{I}(M,-)$ from the category $R$-modules to itself. Let $L_{i} \Lambda_{I}(M,-)$ be the $i$ th left derived functor of $\Lambda_{I}(M,-)$. The $i$ th generalised local homology module $H_{i}^{I}(M, N)$ of $M, N$ with respect to $I$ is defined by (see [12])

$$
H_{i}^{I}(M, N)=\underbrace{\lim }_{t} \operatorname{Tor}_{i}^{R}\left(M / I^{t} M, N\right) .
$$

This definition of generalised local homology modules is in some sense dual to the definition of generalised local cohomology modules of Herzog [5] and in fact a generalisation of the usual local homology $H_{i}^{I}(M)=\lim _{t} \operatorname{Tor}_{i}^{R}\left(R / I^{t}, M\right)$. In [10] we also studied some basic properties of the left derived functor $L_{i} \Lambda_{I}(M,-)$ of $\Lambda_{I}(M,-)$

This research is funded by Vietnam National Foundation for Science and Technology Development (NAFOSTED) under grant number 101.01-2011.24.

(C) 2012 Australian Mathematical Publishing Association Inc. 0004-9727/2012 \$16.00 
and showed that if $M$ is a finitely generated $R$-module and $N$ a linearly compact $R$-module, then $L_{i} \Lambda_{I}(M, N) \cong H_{i}^{I}(M, N)$ for all $i \geq 0$. However, the nonvanishing of the modules $L_{i} \Lambda_{I}(M, N)$ is a rather difficult problem. In Section 2, Theorem 2.1 gives us equivalent statements for vanishing and nonvanishing of the modules $L_{i} \Lambda_{I}(M, N)$. In Theorem 2.5 we study the set of co-associated primes of the modules $L_{i} \Lambda_{I}(M, N)$ and show that if $M$ is a finitely generated $R$-module and $N$ an $R$-module with $l_{+}^{I}(N)=d$ and $r=\operatorname{pd}(M)<\infty$, then there is submodule $X$ of $\operatorname{Tor}_{r}^{R}\left(M ; L_{d-1} \Lambda_{I}(N)\right)$ such that

$$
\operatorname{Coass}\left(L_{d+r-1} \Lambda_{I}(M ; N)\right) \subseteq \operatorname{Coass}\left(\operatorname{Tor}_{r-1}^{R}\left(M ; L_{d} \Lambda_{I}(N)\right)\right) \cup \operatorname{Coass}(X) .
$$

It should be mentioned that when $M$ is a finitely generated $R$-module, the left derived functors $L_{i} \Lambda_{I}(M,-)$ and the generalised local homology functors $H_{i}^{I}(M,-)$ are coincident on the category of linearly compact $R$-modules. In the final section we study the co-localisation of generalised local homology modules $H_{i}^{I}(M, M)$ when $N$ is a semi-discrete linearly compact $R$-module and prove that if $r=\operatorname{pd}(M)<\infty$ and $\operatorname{Ndim} N=d$, then $\operatorname{Cosupp}_{R}\left(H_{d+r}^{I}(M ; N)\right) \subseteq\{m\}$ (Theorem 3.3). Note that $\operatorname{Ndim} M$ is the noetherian dimension defined by Roberts [14] (see also [6]). It should be mentioned that the class of linearly compact modules is large, containing important classes of modules. Even its subclass of semi-discrete linearly compact modules contains artinian modules, as well as finitely generated modules over a complete ring. Further information on linearly compact modules can be found in [7] or [2].

\section{The top left derived functors of the generalised $I$-adic completion}

For two $R$-modules $M$ and $N$, we put

$$
\begin{gathered}
\operatorname{tor}_{+}(M, N)=\sup \left\{i \mid \operatorname{Tor}_{i}^{R}(M, N) \neq 0\right\}, \\
l_{+}^{I}(M, N)=\sup \left\{i \mid L_{i} \Lambda_{I}(M, N) \neq 0\right\}
\end{gathered}
$$

and

$$
l_{+}^{I}(N)=\sup \left\{i \mid L_{i} \Lambda_{I}(N) \neq 0\right\} .
$$

Theorem 2.1. Let $M$ be a finitely generated $R$-module and $N$ an $R$-module such that $r=\operatorname{pd}(M)<\infty$ and $d=l_{+}^{I}(N)<\infty$. Then the following statements are equivalent:

(i) $\quad r=\operatorname{tor}_{+}\left(M, L_{d} \Lambda_{I}(N)\right)$;

(ii) $L_{r+d} \Lambda_{I}(M, N) \neq 0$;

(iii) $l_{+}^{I}(M, N)=r+d$.

To prove Theorem 2.1 we need the following lemmas.

Lemma 2.2 [11, Lemma 2.5]. Let $M$ be a finitely generated $R$-module and $F$ a free R-module. Then

$$
M \otimes_{R} \Lambda_{I}(F) \cong \Lambda_{I}(M, F) .
$$

Lemma 2.3. Let $M$ be a finitely generated $R$-module and $N$ an $R$-module. If $\operatorname{Tor}_{p}^{R}\left(M ; L_{q} \Lambda_{I}(N)\right)=0$ for all $p>\operatorname{ror} q>d$, then

$$
\operatorname{Tor}_{r}^{R}\left(M ; L_{d} \Lambda_{I}(N)\right) \cong L_{r+d} \Lambda_{I}(M, N) .
$$


Proof. Let us consider functors $F=M \otimes_{R^{-}}$and $G=\Lambda_{I}$. The functor $F$ is obviously right exact. On the other hand, it follows from [1, Theorem 1.4.7] that a projective module $P$ implies $\Lambda_{I}(P)$ is flat and then is $F$-acyclic. Hence, combining [15, Theorem 11.39] with Lemma 2.2 yields a Grothendieck spectral sequence

$$
E_{p, q}^{2}=\operatorname{Tor}_{p}^{R}\left(M, L_{q} \Lambda_{I}(N)\right) \underset{p}{\rightarrow} L_{p+q} \Lambda_{I}(M, N) .
$$

Thus there is a filtration $\Phi$ of $L_{p+q} \Lambda_{I}(M, N)$ with

$$
0=\Phi^{-1} H_{r+d} \subseteq \cdots \subseteq \Phi^{r+d} H_{r+d}=L_{r+d} \Lambda_{I}(M, N)
$$

and

$$
E_{p, r+d-p}^{\infty} \cong \Phi^{p} H_{r+d} / \Phi^{p-1} H_{r+d}, \quad 0 \leq p \leq r+d .
$$

As $\operatorname{Tor}_{p}^{R}\left(M ; L_{q} \Lambda_{I}(N)\right)=0$ for all $p>r$ or $q>d, E_{p, r+d-p}^{2}=0$ for all $p \neq r$.

We have

$$
\Phi^{r-1} H_{r+d}=\Phi^{r-2} H_{r+d}=\cdots=\Phi^{-1} H_{r+d}=0
$$

and

$$
\Phi^{r} H_{r+d}=\Phi^{r+1} H_{r+d}=\cdots=\Phi^{r+d} H_{r+d}=L_{r+d} \Lambda_{I}(M, N) .
$$

It follows that $\Phi^{r} H_{r+d} \cong E_{r, d}^{\infty}$, which means that $L_{r+d} \Lambda_{I}(M, N) \cong E_{r, d}^{\infty}$. To finish the proof we consider homomorphisms of the spectral sequence

$$
E_{r+k, d-k+1}^{k} \longrightarrow E_{r, d}^{k} \longrightarrow E_{r-k, d+k-1}^{k} \text {. }
$$

The hypothesis gives $E_{r+k, d-k+1}^{k}=E_{r-k, d+k-1}^{k}=0$ for all $k \geq 2$. Therefore

$$
\operatorname{Tor}_{r}^{R}\left(M, L_{d} \Lambda_{I}(N)\right)=E_{r, d}^{2}=E_{r, d}^{3}=\cdots=E_{r, d}^{\infty} \cong L_{r+d} \Lambda_{I}(M, N) .
$$

The proof is complete.

Corollary 2.4. Let $M$ be a finitely generated $R$-module and $N$ an $R$-module such that $r=\operatorname{pd}(M)<\infty$ and $d=l_{+}^{I}(N)<\infty$. Then

$$
\operatorname{Tor}_{r}^{R}\left(M ; L_{d} \Lambda_{I}(N)\right) \cong L_{d+r} \Lambda_{I}(M ; N) \text {. }
$$

We are now in a position to prove Theorem 2.1.

Proof of Theorem 2.1. (i) $\Rightarrow$ (ii) By Corollary 2.4, we have the isomorphism

$$
\operatorname{Tor}_{r}^{R}\left(M ; L_{d} \Lambda_{I}(N)\right) \cong L_{r+d} \Lambda_{I}(M ; N) .
$$

As $r=$ tor $_{+}\left(M, L_{d} \Lambda_{I}(N)\right.$, we get $L_{r+d} \Lambda_{I}(M, N) \neq 0$.

(ii) $\Rightarrow$ (iii) Assume that $L_{r+d} \Lambda_{I}(M, N) \neq 0$. For all $j>d+r$, we have

$$
\operatorname{Tor}_{r}^{R}\left(M ; L_{j-r} \Lambda_{I}(N)\right) \cong L_{j} \Lambda_{I}(M ; N)
$$

by Lemma 2.3. It follows that $L_{j} \Lambda_{I}(M ; N)=0$ and then that $l_{+}^{I}(M, N)=r+d$. 
(iii) $\Rightarrow$ (i) We have $\operatorname{Tor}_{r}^{R}\left(M ; L_{d} \Lambda_{I}(N)\right) \cong L_{d+r} \Lambda_{I}(M ; N) \neq 0$. For all $i>r$,

$$
\operatorname{Tor}_{i}^{R}\left(M ; L_{d} \Lambda_{I}(N)\right) \cong L_{i+d} \Lambda_{I}(M ; N)=0,
$$

as $l_{+}^{I}(M, N)=r+d$. Therefore $r=\operatorname{tor}_{+}\left(M, L_{d} \Lambda_{I}(N)\right)$.

A prime ideal $\mathfrak{p}$ is said to be co-associated to a nonzero $R$-module $M$ if there is an artinian homomorphic image $T$ of $M$ with $\mathfrak{p}=\mathrm{Ann}_{R} T$. The set of co-associated primes of $M$ is denoted by $\operatorname{Coass}_{R}(M)$. It should be noted that if $M$ is a semi-discrete linearly compact $R$-module, then the set $\operatorname{Coass}_{R}(M)$ is finite [18, Property 1(L4)].

If $0 \longrightarrow N \longrightarrow M \longrightarrow K \longrightarrow 0$ is an exact sequence of $R$-modules, then $\operatorname{Coass}_{R}(K) \subseteq$ $\operatorname{Coass}_{R}(M) \subseteq \operatorname{Coass}_{R}(N) \cup \operatorname{Coass}_{R}(K)$ [17, Theorem 1.10].

THEOREM 2.5. Let $M$ be a finitely generated $R$-module and $N$ an $R$-module. If $l_{+}^{I}(N)=$ $d$ and $r=\operatorname{pd}(M)<\infty$, then there is a submodule $X$ of $\operatorname{Tor}_{r}^{R}\left(M ; L_{d-1} \Lambda_{I}(N)\right)$ such that

$$
\operatorname{Coass}\left(L_{d+r-1} \Lambda_{I}(M ; N)\right) \subseteq \operatorname{Coass}\left(\operatorname{Tor}_{r-1}^{R}\left(M ; L_{d} \Lambda_{I}(N)\right)\right) \cup \operatorname{Coass}(X) .
$$

Proof. We have the Grothendieck spectral sequence

$$
E_{p, q}^{2}=\operatorname{Tor}_{p}^{R}\left(M ; L_{q} \Lambda_{I}(N)\right) \underset{p}{\rightarrow} L_{p+q} \Lambda_{I}(M ; N) .
$$

Then there is a filtration $\Phi$ of $L_{p+q} \Lambda_{I}(M ; N)$ with

$$
0=\Phi^{-1} H_{d+r-1} \subseteq \cdots \subseteq \Phi^{d+r-1} H_{d+r-1}=L_{d+r-1} \Lambda_{I}(M ; N)
$$

and

$$
E_{p, d+r-1-p}^{\infty}=\Phi^{p} H_{d+r-1} / \Phi^{p-1} H_{d+r-1}, \quad 0 \leq p \leq d+r-1 .
$$

Note that $L_{q} \Lambda_{I}(N)=0$ for all $q>d$, so $E_{p, d+r-1-p}^{2}=0$ for all $p \neq r, r-1$. Thus

$$
\Phi^{r} H_{d+r-1}=\Phi^{r+1} H_{d+r-1}=\cdots=\Phi^{d+r-1} H_{d+r-1}=L_{d+r-1} \Lambda_{I}(M ; N)
$$

and

$$
\Phi^{r-2} H_{d+r-1}=\Phi^{r-3} H_{d+r-1}=\cdots=\Phi^{-1} H_{d+r-1}=0 .
$$

It follows that

$$
\Phi^{r-1} H_{d+r-1} \cong E_{r-1, d}^{\infty} \quad \text { and } \quad E_{r, d-1}^{\infty} \cong L_{d+r-1} \Lambda_{I}(M ; N) / \Phi^{r-1} H_{d+r-1} .
$$

We now consider homomorphisms of the spectral sequence

$$
\begin{gathered}
E_{r-1+k, d-k+1}^{k} \longrightarrow E_{r-1, d}^{k} \longrightarrow E_{r-1-k, d+k-1}^{k}, \\
E_{r+k, d-k}^{k} \longrightarrow E_{r, d-1}^{k} \longrightarrow E_{r-k, d+k-2}^{k} .
\end{gathered}
$$


As $E_{r-1+k, d-k+1}^{k}=E_{r-1-k, d+k-1}^{k}=E_{r+k, d-k}^{k}=0$ for all $k \geq 2$ and $E_{r-k, d+k-2}^{k}=0$ for all $k \geq 3$,

$$
\operatorname{Tor}_{r-1}^{R}\left(M ; L_{d} \Lambda_{I}(N)\right)=E_{r-1, d}^{2}=E_{r-1, d}^{3}=\cdots=E_{r-1, d}^{\infty}
$$

and $E_{r, d-1}^{3}=E_{r, d-1}^{4}=\cdots=E_{r, d-1}^{\infty}$, a submodule of $E_{r, d-1}^{2}$. Setting $X=E_{r, d-1}^{\infty}$, we have a short exact sequence

$$
0 \longrightarrow \operatorname{Tor}_{r-1}^{R}\left(M ; L_{d} \Lambda_{I}(N)\right) \longrightarrow L_{d+r-1} \Lambda_{I}(M ; N) \longrightarrow X \longrightarrow 0
$$

which finishes the proof.

\section{Co-support of local homology modules}

In this section we study the the generalised local homology functors $H_{i}^{I}(M,-)$ when $M$ is a finitely generated $R$-module. Note that the left derived functors $L_{i} \Lambda_{I}(M,-)$ and the generalised local homology functors $H_{i}^{I}(M,-)$ are coincident on the category of linearly compact $R$-modules.

Lemma 3.1 [2, Proposition 3.5] and [10, Theorem 3.6]. Let $M$ be a finitely generated $R$-module and $N$ a linearly compact $R$-module. Then:

(i) $L_{i} \Lambda_{I}(N) \cong H_{i}^{I}(N)$ for all $i \geq 0$;

(ii) $L_{i} \Lambda_{I}(M, N) \cong H_{i}^{I}(M, N)$ for all $i \geq 0$.

Let $S$ be a multiplicative subset of $R$. Following [9], the co-localisation of an $R$ module $M$ with respect to $S$ is the module ${ }_{S} M=\operatorname{Hom}\left(R_{S}, M\right)$. If $M$ is a linearly compact $R$-module, then ${ }_{S} M$ is also a linearly compact $R$-module by [2, Lemma 2.5]. If $M$ is an artinian $R$-module, then ${ }_{S} M$ is not necessarily artinian (see [9, Section 4]) but is a linearly compact $R$-module. Let $\mathfrak{p}$ be a prime of $R$ and $S=R-\{\mathfrak{p}\}$; then instead of ${ }_{S} M$ we write ${ }_{p} M$.

For an $R$-module $M$, Melkersson and Schenzel [9] defined the co-support of $M$ to be the set

$$
\operatorname{Cos}_{R}(M)=\left\{\left.\mathfrak{p} \in \operatorname{Spec}(R)\right|_{\mathfrak{p}} M \neq 0\right\} .
$$

In [17, Definition 2.1] Yassemi defined the co-support $\operatorname{Cosupp}_{R}(M)$ of an $R$-module $M$ to be the set of primes $\mathfrak{p}$ such that there exists a cocyclic homomorphic image $L$ of $M$ with $\operatorname{Ann}(L) \subseteq \mathfrak{p}$. Note that a module is cocyclic if it is a submodule of $E(R / \mathrm{m})$ for some maximal ideal $\mathrm{m} \in R$. We have $\operatorname{Cos}_{R}(M) \subseteq \operatorname{Cosupp}_{R}(M)$, but the equation is in general not true (see [17, Theorem 2.6]). If $M$ is a linearly compact $R$-module, we proved that $\operatorname{Coass}_{R}(M) \subseteq \operatorname{Cos}_{R}(M)=\operatorname{Cosupp}_{R}(M)$ and every minimal element of $\operatorname{Cosupp}_{R}(M)$ belongs to $\operatorname{Coass}_{R}(M)$ [13, Theorem 3.8] and [4, Theorem 4.2].

The following lemma is used to prove Theorem 3.3.

Lemma 3.2. Let $(R, \mathrm{~m})$ be a local ring and $M$ a finitely generated $R$-module. If $N$ is a linearly compact $R$-module, then for all $i, j \geq 0$ :

(i) $\operatorname{Tor}_{i}^{R}(M ; N) \cong \operatorname{Tor}_{i}^{\hat{R}}(\hat{M} ; N)$ and, especially, $\operatorname{Tor}_{i}^{\hat{R}}\left(\hat{M} ; H_{j}^{I}(N)\right) \cong \operatorname{Tor}_{i}^{R}\left(M ; H_{j}^{I}(N)\right)$;

(ii) $H_{i}^{I \hat{R}}(\hat{M} ; N) \cong H_{i}^{I}(M ; N)$. 
Proof. (i) It follows from [2, Lemma 7.1] that $N$ has a natural linearly compact module structure over $\widehat{R}$. As $\widehat{R}$ is a flat $R$-module, we have, by [15, Theorem 11.53],

$$
\begin{aligned}
\operatorname{Tor}_{i}^{R}(M ; N) & \cong \operatorname{Tor}_{i}^{R}\left(M ; \widehat{R} \otimes_{\hat{R}} N\right) \\
& \cong \operatorname{Tor}_{i}^{\hat{R}}\left(M \otimes_{R} \widehat{R} ; N\right) \cong \operatorname{Tor}_{i}^{\hat{R}}(\hat{M} ; N) .
\end{aligned}
$$

The second statement is an immediate consequence, since $H_{j}^{I}(N)$ is linearly compact by [2, Proposition 3.3].

(ii) For all $i \geq 0, t>0$, by (i),

$$
\operatorname{Tor}_{i}^{R}\left(M / I^{t} M ; N\right) \cong \operatorname{Tor}_{i}^{\hat{R}}\left(\hat{M} /(I \hat{R})^{t} \hat{M} ; N\right) .
$$

Passing to inverse limits, we have the isomorphism as required.

We now recall the concept of noetherian dimension of an $R$-module $M$, denoted by $\operatorname{Ndim} M$. Note that the notion of noetherian dimension was introduced first by Roberts [14] under the term 'Krull dimension'. Kirby [6] later changed Roberts's terminology and referred to noetherian dimension to avoid confusion with the wellknown Krull dimension of finitely generated modules. Let $M$ be an $R$-module. When $M=0$ we put $\operatorname{Ndim} M=-1$. Then by induction, for any ordinal $\alpha$, we put $\operatorname{Ndim} M=\alpha$ when (i) $\operatorname{Ndim} M<\alpha$ is false, and (ii) for every ascending chain $M_{0} \subseteq M_{1} \subseteq \cdots$ of submodules of $M$, there exists a positive integer $m_{0}$ such that $\operatorname{Ndim}\left(M_{m+1} / M_{m}\right)<\alpha$ for all $m \geq m_{0}$. Thus $M$ is nonzero and finitely generated if and only if $\operatorname{Ndim} M=0$. If $0 \longrightarrow M^{\prime \prime} \longrightarrow M \longrightarrow M^{\prime} \longrightarrow 0$ is a short exact sequence of $R$-modules, then $\operatorname{Ndim} M=\max \left\{\operatorname{Ndim} M^{\prime \prime}, \operatorname{Ndim} M^{\prime}\right\}$. For each subset $B$ of $R$, let $V(B)$ denote the set of all primes of $R$ which contain $B$.

THEOREM 3.3. Let $(R, \mathrm{~m})$ be a local ring of dimension $d$ and $N$ a semi-discrete linearly compact $R$-module.

(i) If $\operatorname{dim} R=d$, then

(a) $\operatorname{Cosupp}_{R}\left(H_{d}^{I}(N)\right) \subseteq\{\mathrm{m}\}$;

(b) $\operatorname{Cosupp}_{R}\left(H_{d-1}^{I}(N)\right)$ is finite.

(ii) If $M$ is a finitely generated R-module with $r=\operatorname{pd}(M)<\infty$, and $\operatorname{Ndim} N=d$, then $\operatorname{Cosupp}_{R}\left(H_{d+r}^{I}(M ; N)\right) \subseteq\{\mathrm{m}\}$.

Proof. (i) (a) Let us first give a proof in the special case where $N$ is artinian. From [17, Proposition 2.3], $\operatorname{Cosupp}_{R}(N)=V\left(\operatorname{Ann}_{R}(N)\right)$. It should be noted that, by [16, Lemma 1.11], $N$ has a natural artinian module structure over $\widehat{R}$ and the going-down theorem holds for the canonical $R \longrightarrow \widehat{R}$. Therefore we may assume that $(R, \mathrm{~m})$ is complete by Lemma 3.2. We mention that $d=\operatorname{dim} R \geq \operatorname{Ndim} N$. If $d>\operatorname{Ndim} N$, then $H_{d}^{I}(N)=0$ because of [2, Theorem 4.8]. We need only give a proof when $d=\operatorname{Ndim} N$. Note that $H_{d}^{I}(N)$ is finitely generated by [2, Theorem 5.3]. From [17, Theorem 2.10] we get $\operatorname{Cosupp}\left(H_{d}^{I}(N)\right) \subseteq\{\mathfrak{m}\}$.

We now turn to the case where $M$ is semi-discrete linearly compact. By [2, Corollary 4.5], there is an isomorphism $H_{i}^{I}(N) \cong H_{i}^{I}\left(\Gamma_{\mathfrak{m}}(N)\right)$ for all $i \geq 1$ and $\Gamma_{\mathfrak{m}}(N)$ 
is artinian. So the lemma is true for $d \geq 1$. When $d=0$, there is, from [18, Theorem], a short exact sequence $0 \longrightarrow B \longrightarrow N \longrightarrow A \longrightarrow 0$ where $A$ is artinian and $B$ is finitely generated. It induces an exact sequence $H_{0}^{I}(B) \longrightarrow H_{0}^{I}(N) \longrightarrow H_{0}^{I}(A) \longrightarrow 0$. According to the above proof, we have $\operatorname{Cosupp}\left(H_{0}^{I}(A)\right) \subseteq\{\mathfrak{m}\}$. On the other hand, combining [2, Corollary 3.11] with [17, Theorem 2.10] gives $\operatorname{Cosupp}\left(H_{0}^{I}(B)\right)=\operatorname{Cosupp}(B)=\{\mathfrak{m}\}$. This finishes the proof of (a).

(b) We first deal with the special case where $N$ is artinian. By an analysis similar to that in the proof of (i), we may assume that $(R, \mathfrak{m})$ is complete. Let $D(N)$ denote the Matlis dual of $N$. We have $H_{d-1}^{I}(N) \cong D\left(H_{I}^{d-1}(D(N))\right)$ by [3, Proposition 3.3]. Applying [17, Corollary 2.9] yields

$$
\begin{aligned}
\operatorname{Cosupp}_{R}\left(H_{d-1}^{I}(N)\right) & =\operatorname{Cosupp}_{R}\left(D\left(H_{I}^{d-1}(D(N))\right)\right) \\
& =\operatorname{Supp}_{R}\left(H_{I}^{d-1}(D(N))\right) .
\end{aligned}
$$

The last set is finite by [8, Theorem 2.4] and so $\operatorname{Cosupp}_{R}\left(H_{d-1}^{I}(N)\right)$ is finite.

We can now proceed analogously to the proof of (a) for the case where $N$ is semidiscrete linearly compact and the proof of (b) is complete.

(ii) From [2, Theorem 4.8], $H_{i}^{I}(N)=0$ for all $i>d$. Thus, combining Lemmas 2.3 and 3.1 yields $H_{d+r}^{I}(M ; N) \cong \operatorname{Tor}_{r}^{R}\left(M ; H_{d}^{I}(N)\right)$. As $M$ is finitely generated, there is a free resolution of $M$ with finitely many free modules. Then $\operatorname{Tor}_{r}^{R}\left(M ; H_{d}^{I}(N)\right)$ is isomorphic to a subquotient of a finite direct sum of copies of $H_{d}^{I}(N)$. Therefore $\operatorname{Cosupp}_{R}\left(\operatorname{Tor}_{r}^{R}\left(M ; H_{d}^{I}(N)\right)\right) \subseteq \operatorname{Cosupp}_{R}\left(H_{d}^{I}(N)\right)$ and the conclusion follows from (i).

\section{References}

[1] J. Bartijn, Flatness, Completions, Regular Sequences, un Ménage à Trois, Thesis, Utrecht, 1985.

[2] N. T. Cuong and T. T. Nam, 'A local homology theory for linearly compact modules', J. Algebra 319 (2008), 4712-4737.

[3] N. T. Cuong and T. T. Nam, 'The $I$-adic completion and local homology for Artinian modules', Math. Proc. Cambridge Philos. Soc. 131 (2001), 61-72.

[4] N. T. Cuong and T. T. Nam, 'On the co-localization, co-support and co-associated primes of local homology modules', Vietnam J. Math. 29(4) (2001), 359-368.

[5] J. Herzog, Komplexe, Auflösungen und Dualität in der localen Algebra (Habilitationsschrift Univ. Regensburg, 1970).

[6] D. Kirby, 'Dimension and length of artinian modules', Quart. J. Math. Oxford 2(41) (1990), 419-429.

[7] I. G. Macdonald, 'Duality over complete local rings', Topology 1 (1962), 213-235.

[8] T. Marley, 'The associated primes of local cohomology modules over rings of small dimension', Manuscripta Math. 104 (2001), 519-525.

[9] L. Melkersson and P. Schenzel, 'The co-localization of an Artinian module', Proc. Edinb. Math. Soc. 38 (1995), 121-131.

[10] T. T. Nam, 'Left derived functors od the generalized $I$-adic completion and generalized local homology', Comm. Algebra 38 (2010), 440-453.

[11] T. T. Nam, 'The finiteness of coassociated primes of generalized local homology modules', Preprint.

[12] T. T. Nam, 'Generalized local homology and cohomology for artinian modules', Algebra Colloquium 19(1) (2012), 1205-1212.

[13] T. T. Nam, 'Co-support and Coartinian modules', Algebra Colloq. 15(1) (2008), 83-96. 
[14] R. N. Roberts, 'Krull dimension for artinian modules over quasilocal commutative rings', Quart. J. Math. Oxford 26(3) (1975), 269-273.

[15] J. J. Rotman, An Introduction to Homological Algebra (Academic Press, 1979).

[16] R. Y. Sharp, 'A method for the study of artinian modules with an application to asymptotic behavior', Commutative Algebra, Math. Sciences Research Inst. Publ., 15 (Springer, 1989), pp. 443-465.

[17] S. Yassemi, 'Coassociated primes', Comm. Algebra 23 (1995), 1473-1498.

[18] H. Zöschinger, 'Linear-kompakte Moduln über noetherschen Ringen', Arch. Math. 41 (1983), 121-130.

TRAN TUAN NAM, Department of Mathematics - Informatics,

Ho Chi Minh University of Pedagogy, 280 An Duong Vuong, District 5,

Ho Chi Minh City, Vietnam

e-mail: namtuantran@gmail.com 